\title{
OPTIMAL SAMPLING OF ELECTROPHYSIOLOGICAL SIGNALS
}

Fu-Shan Jaw

Institute of Biomedical Engineering, National Taiwan University, Taipei, Taiwan

(Accepted January 10, 2001)

\section{SUMMARY}

Action potentials (APs) and field potentials (FPs) are commonly used to study functions of the brain. To facilitate their analysis, computer methods are often used. The high-speed nature of APs and the large amount of data of FPs put stringent requirements on the sampling rate used. To determine optimal sampling rates for these signals a modified sampling equation is derived. This equation formulates the relationship among the order of the anti-aliasing filter, the sampling rate, and the number of bits of quantization. For convenience, a table is also provided to reveal the optimal sampling rate for various combinations of filters and analog-to-digital converter cards commonly available. The recordings of compound action potentials, APs, and FPs were practically used to further examine this relationship. Under the specified conditions for the recording of thesc electrophysiological signals, their highest frequency contents are about $10 \mathrm{kHz}, 9.3 \mathrm{kHz}$ and $40 \mathrm{~Hz}$, respectively. In conclusion, this paper provides a tutorial, quick reference, and step-by-step guide to sample a signal faithfully; that is, free from the contamination of aliasing errors no matter the spectrum of the signal is known. Also, the role and necessity of a high order anti-aliasing filter is emphasized.

KEYWORDS: analog-to-digital conversion; data acquisition; action potential; field potential; alias

\section{INTRODUCTION}

Computers are commonly used in electrophysiology for data acquisition and signal processing. However, after digitization, the data amount of ficld potentials (FPs) and action potentials (APs) is usually large. For a three dimensional mapping of FPs in a small region of $2 \times 2 \times 1.5 \mathrm{~mm}$, the number of recording points could be greater than 48,000 if the spatial resolution is $50 \mu \mathrm{m}(1,2)$. Moreover, at each recording point a total of 50 cycles of responses was recorded for signal averaging (3). A high sampling rate is mandatory for the acquisition of APs due to their high frequency 
contents $(4,5)$. Both the high-speed nature of APs and many recording points of FPs put stringent requirements on the sampling rates used.

In theory, a signal can be represented by its samples if the sampling rate is greater than twice the highest frequency present in the signal (6). In practice, due to the finite roll-off rate of an anti-aliasing filter one usually needs to sampled more times than this Nyquist rate to get a reasonably faithful waveform from its samples $(7,8)$. However, practical criteria or guidelines about how to digitize these signals optimally are still not so clear, because not all the physiologists are familiar with details of $\mathrm{A} / \mathrm{D}$ conversion. To address this question, we derived a formula that make it possible to calculate the sampling frequency in relation to the signal bandwidth, the parameters of an anti-aliasing filter, and the resolution of the A/D converter. Furthermore, to illustrate the aliasing phenomenon in detail compound action potentials (CAPs) of a dorsal root were sampled at different speeds. Finally, some recordings of FPs and APs were presented to perform a practical examination of this relationship. I hope this information could be helpful for other laboratories that deal with the acquisition of electrophysiological signals.

\section{MATERIALS AND METHODS}

\section{Theoretical considerations}

Before data acquisition, the sampling rate, the resolution of digitization, and the order of the anti-aliasing filter should be determined. To find the relationship among these parameters it is assumed that the sampling rate ( $\mathrm{fs}$ ) is $\mathrm{n}$ times the highest frequency content of the signal, which is normalized to $1 \mathrm{~Hz}$ here for simplicity. Also, a p-pole anti-aliasing filter is assumed to be used just prior to the A/D converter. The folding frequency (fo) is defined as a half of the sampling frequency. Since the spectral shapes from fo to fs and from DC to fo are mirror images of each other, a noise at $\mathrm{n}-1 \mathrm{~Hz}$ will contaminate the frequency $1 \mathrm{~Hz}$, the highest frequency component of the signal. We further assume that the noise amplitude at the frequency $\mathrm{n}-1 \mathrm{~Hz}$ is of full-scale magnitude of the input 
range of the $\mathrm{A} / \mathrm{D}$ converter. The noises present at frequency higher than $\mathrm{n}-1 \mathrm{~Hz}$ will be more attenuated by the anti-aliasing filter, and therefore are not considered. As a common practice an acceptable aliasing error is within \pm 1 least significant bit (LSB). To achieve this goal, the amount of attenuation at n-1 Hz should be greater than the dynamic range (1LSB) of the A/D converter.

Based on these assumptions, we can thus obtain the following relationship:

$$
-6 \mathrm{p} \log _{2}(n-1) \leqq-20 \log 2^{\mathrm{b}}
$$

Where $\mathrm{p}$ is the order of the filter, $\mathrm{n}$ the quotient between the sampling rate and the cutoff frequency of the filter, and $b$ is the number of bits of the $A / D$ converter.

The left part of Equation (1) is the remaining aliasing error after filtering whereas the right part is 1 LSB in dB. After some manipulation the relationship becomes:

$$
n \geqq 2^{\mathrm{b} / \mathrm{p}}+1
$$

From the above relationship a quick reference between the sampling rates with the orders of the anti-aliasing filter is summarized in Table 1.

Table 1. Minimum values of $n$ required to limit the aliasing error within $1 \mathrm{LSB}$ with $b$ and $p$ as parameters.

\begin{tabular}{llllllll}
\hline $\mathrm{P}$ & 2 & 3 & 4 & 5 & 6 & 7 & 8 \\
$\mathrm{n}(\mathrm{b}=8)$ & 17 & 7.3 & 5 & 4 & 3.5 & 3.2 & 3 \\
$\mathrm{n}(\mathrm{b}=12)$ & 65 & 17 & 9 & 6.3 & 5 & 4.3 & 3.8 \\
$\mathrm{n}(\mathrm{b}=16)$ & 257 & 41.3 & 17 & 10.2 & 7.3 & 5.9 & 5
\end{tabular}

This table provides an easy guide of selection for who are not familiar with mathematics. As expected, the lower the order of the filter the higher the sampling rate should be used. Also, the higher the dynamic range of digitization, the higher the sampling rate is needed for the same order of the anti-aliasing filter. The transient response of a filter degrades as its order increases. Hence, as a compromise, 5-pole anti-aliasing filters were used in the following experiments and the sampling 
rates of a 12-bit $\mathrm{A} / \mathrm{D}$ conversion were programmed to be greater than 6.3 times the cutoff frequencies of these anti-aliasing filters.

\section{Electrophysiological recordings}

A total of 10 adult Wistar rats were used. The animal preparation was the same as the previous paper (9). Isolated sacral dorsal roots werc supramaximally stimulated to elicit CAPs of the A-fiber. The FPs in the thalamus, which were evoked by the CAPs, were recorded by a glass microelectrode with a large tip diameter of $30 \mu \mathrm{m}(10)$ (impedance $=350 \pm 50 \mathrm{k} \Omega, \mathrm{n}=7$, filled with $3 \mathrm{M} \mathrm{NaCl}$ ). Evoked single-unit APs were recorded extracellularlly at a similar place with conventional glass microelectrodes (impedance $=20 \pm 5 \mathrm{M} \Omega, \mathrm{n}=5,3 \mathrm{M} \mathrm{NaCl}$ ).

The CAPs and APs were amplified differentially with a gain of 50 for CAPs and 2500 for APs. Since the duration of the CAPs and the APs is short the transient response of the anti-aliasing filter is important. Therefore, a 5-pole Bessel low-pass filter with a cutoff frequency of $10 \mathrm{kHz}$ was used because of its linear-phase property. Then, they were digitized by a 12-bit A/D converter at a time interval of $15 \mu \mathrm{s}$. In contrast, FPs are "slow" wave that requires an anti-aliasing filter with maximally flatness in the pass-band. Therefore, FPs were recorded with a gain of 500 and filtered by a 5-pole Butterworth low-pass filter with a bandwidth of $50 \mathrm{~Hz}$. Because the spontaneous and environmental noises are quite large with respect to the magnitude of FPs a total of 100 cycles of FPs were sampled at $350 \mathrm{~Hz}$ for signal averaging. For ease of spectral analysis, a total of 512 data points was sampled per cycle for all the signals.

The sampled signals were imported into a mathematics software (MATLAB) for spectral analysis. Both the waveforms of time domain and frequency domain were exported to a graphic software (Sigmaplot) for data presentation. 


\section{RESULTS}

Sampling CAPs at different speeds: alias

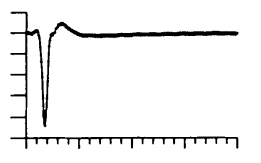

A
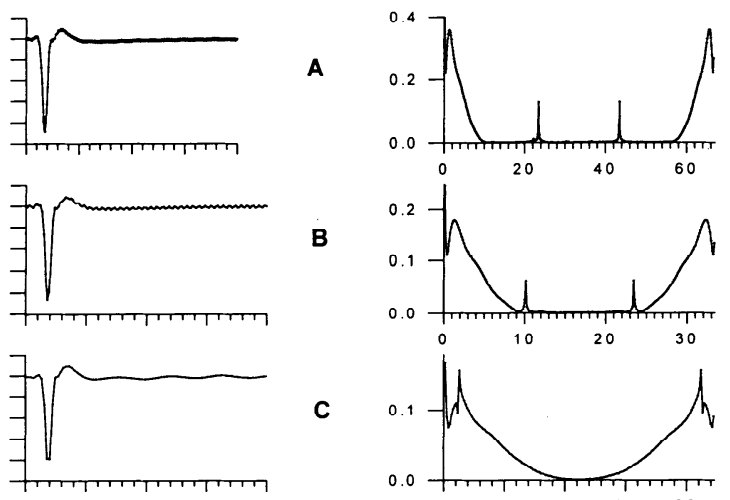

c
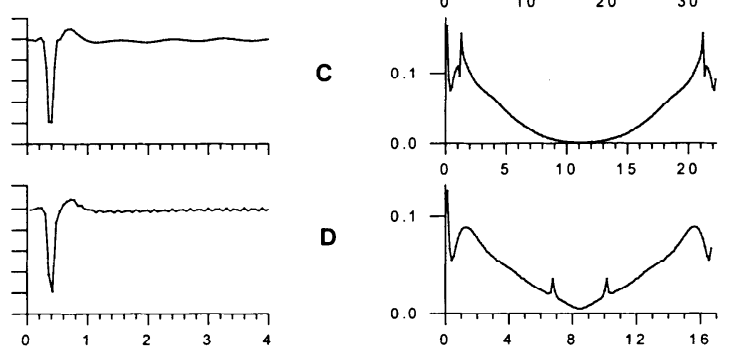

Fig. 1. Effect of sampling rates on the waveform of CAPs. (A) A total of 512 points were sampled in the original CAP. To simulate different sampling rates the data were progressively reduced to 256,171 , and 128 points in (B), (C), and (D), respectively. It is noted that the fewer the points the smaller the relative amplitudes of the spectra. The unit of the abscissa is ms in time domain and $\mathrm{kHz}$ in frequency domain. A vertical scale in the left part of this figure is $0.01 \mathrm{~V}$.

The CAP of the third dorsal root of the sacral cord (S3) was digitized at $66.67 \mathrm{kHz}(15 \mu \mathrm{s})$ in Fig. 1A. A noise appears at $23.43 \mathrm{kHz}$, which may come from the power line. If every other point of the sampled data was used for frequency analysis, the sampling rate becomes $33.33 \mathrm{kHz}$ as shown in Fig. 1B. Here, the noise is undersampled and is reflected onto $10.16 \mathrm{kHz}$; it is the difference between the sampling frequency $(33.33 \mathrm{kHz})$ and the frequency of the noise $(23.43 \mathrm{kHz})$. The frcquency of the reflected noise may not be exactly the same as the difference due to the discrete nature of the discrete Fourier transformation. It is known from Fig. 1A that the upper limit of the 
spectrum is below $10 \mathrm{kHz}$. The noise (either in Fig. 1A or in 1B) does not contaminate the spectrum of the CAP and can be removed completely by the process of digital filtering.

If we further reduce the sampling rate to $22.22 \mathrm{kHz}$ by selecting every third point of the original data as shown in Fig. 1C. An aliasing error caused by undersampling appears at $1.30 \mathrm{kHz}$. In a similar manner $16.67 \mathrm{kHz}$ is obtained by every fourth point and will produce an alias at $6.77 \mathrm{kHz}$ as shown in Fig. 1D. Unfortunately, both the alias errors in Fig. 1C and 1D cannot be filtered out without affecting the waveform of the CAP due to overlapping in spectra. The aliasing error depends not on the frequency of the noise but on the difference between the noise and the folding frequencies (fo=1/2 fs). In Fig. 1C (especially the time domain portion) it appears at a lower frequency although its sampling rate is higher while compared with that of Fig. 1D.

\section{Frequency spectra of FPs and APs}

As recorded by the specified method a representative waveform of the evoked FPs is shown in the left portion of Fig. 2. It is clear from Fig. $2 \mathrm{C}$ that there is no frequency component above $35 \mathrm{~Hz}$. We, thus, low-pass filtered the FPs below $35 \mathrm{~Hz}$ by using the inverse partial fast Fourier transform (IFFT). The result is shown in Fig. 2B. Since the area index is more vulnerable than the amplitude one while comparing two similar waveforms (9), the absolute values of difference between the waveforms before and after filtering were integrated as the error index. In this example this index is smaller than $2 \%$.

We also set $2 \%$ of variation in waveform area as the criterion for deciding the upper limit of the frequency content of APs. The value so obtained is $9.26 \pm 0.47 \mathrm{kHz}(\mathrm{n}=5)$. An example of the APs is shown in the right portion of Fig. 2. In this case it is low-pass filtered at $9.1 \mathrm{kHz}$ by utilizing the same method of IFFT. The filtered AP in Fig. 2B posses all the characteristics of the original AP before filtering. 


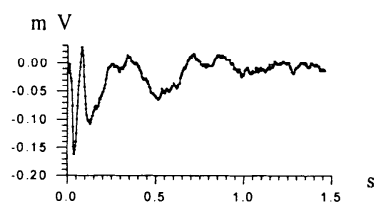

A
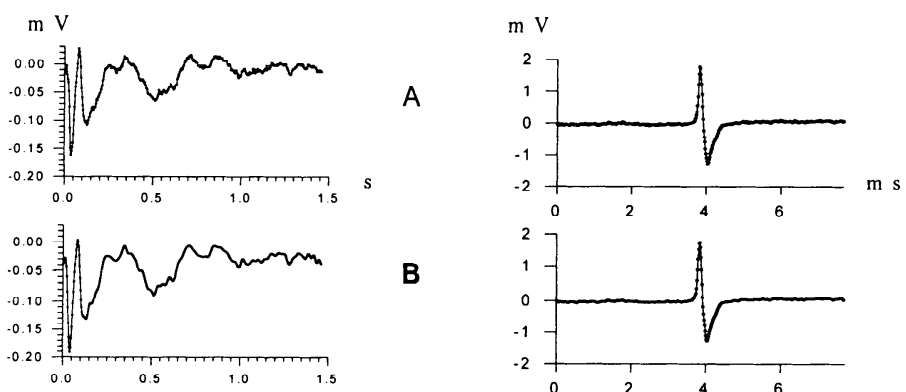

B
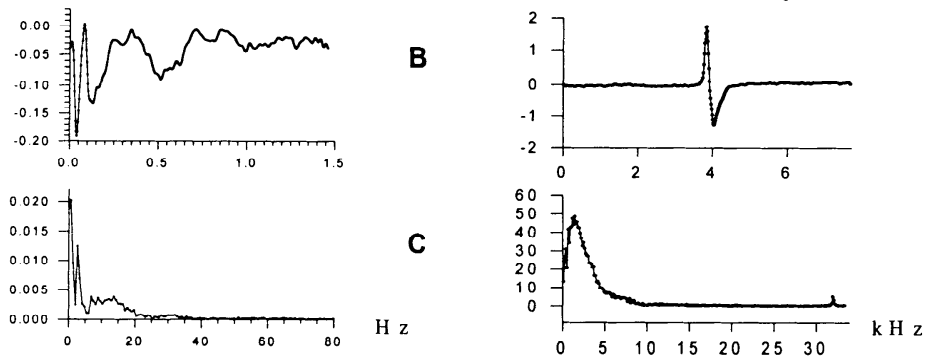

Fig. 2. Waveforms and frequency spectra of an averaged FP (left side) and an AP (right side). (A) Their original waveforms. (B) Effects of low-pass filtering on the waveforms. (C) Their spectral distribution.

\section{DISCUSSION}

From Equation (2) or Table 1 we know that one can sample a signal at a rate from 3 (8-pole filter, 8-bit A/D) to 10 (5-pole, 16 bits) times the highest frequency content of the signal only if a high-order anti-aliasing filter is employed. Two cases in Table 1 are of special interest. A 2-pole low-pass filter, which is often built in amplifiers, and a 12-bit A/D card are possibly the most popular devices. A surprising fact is that with such a combination an impractical high sampling rate, "65 times," should be used. On the other hand, for an 8-pole filter "5 times" is adequate to achieve the dynamic range of 16 bits. Clearly, a filter of high order should be used for limiting the sampling rate and hence the space for data storage within a reasonable value. As shown in Equation (2), 
while the order of the filter increases to infinity the sampling rate can be slowed down to 2 . Thus, Equation (2) can be considered as a modified sampling theorem that takes non-ideal properties of band-limiting filters into account. The importance of a high-order anti-aliasing filter cannot be overemphasized. One could possibly say that the signal he or she deals with is very low frequency in nature. However, the key point is not whether the frequency of the signal is high or not but is the recording bandwidth just prior sampling. If a high frequency noise is present in the recording, the aliasing error might spuriously happen at any frequency of interest even a signal of very low frequency is sampled (11). The purpose of the high-order anti-aliasing filter is not only to limit the bandwidth of a recording but also to suppress noises outside this band at a rate of "sharp enough." Furthermore, the role of the anti-aliasing filter cannot be replaced by the processing of digital filters. The signal should be band-limited just before digitization (6); that is, an analog filter is mandatory for anti-aliasing purpose.

The other important issue about digitization is the error caused by quantization. How many bits does it require for an application depends on the characteristics of the signal, such as the signal-to-noise $(\mathrm{S} / \mathrm{N})$ ratio, the dynamic range, ...etc. For instance, the $\mathrm{S} / \mathrm{N}$ ratio of an extracellular recording is usually not very high due to baseline wandering and the interferences from other spontaneous activities. Considering the fact that the $\mathrm{S} / \mathrm{N}$ ration of a conventional tape recorder is usually lower than $48 \mathrm{~dB}(12)$, an 8-bit $\mathrm{A} / \mathrm{D}$ card maybe adequate for this purpose. On the other hand, the peak amplitude of CAPs of the myelinated fibers in a peripheral nerve is a hundred times larger than those of the unmyelinated fibers (13). A minimum of 12-bit resolution is thus necessary for the sampling of CAPs of a peripheral nerve.

Both from theoretical or from experimental results show that for a 5-pole anti-aliasing filter together with a 12-bit $A / D$, the sampling rate can be set at 6.3 times of the cutoff frequency of the filtcr. This value is less than a half of the 14 times suggested previously (14). It is partially due to the fact that the cutoff frequency of the filter had been moved down to the high-frequency margin of 
the signal. There are several reasons for the justification of doing so. Firstly, the high frequency harmonics of these signals are usually very small and are not necessary in phase. They do not add algebraically in time domain and will contribute very little to the overall waveform. Secondly, a physical filter cannot have an infinite roll-off rate like the partial IFFT does. Thus, distortion caused by an analog anti-aliasing filter should be much lower than the value calculated by the IFFT method. Moreover, in practical environments the noises near the frequency $\mathrm{n}-1 \mathrm{~Hz}$ are seldom so large as assumed previously. Therefore, the value in Table 1 will suggest a safety sampling rate for practical applications.

CAPs, APs, and FPs are possibly the most common signals in electrophysiological laboratories. Their highest frequency components are about $10 \mathrm{kHz}, 9.3 \mathrm{kHz}$, and $40 \mathrm{~Hz}$, respectively. (Note that the frequency spectrum of the CAPs may depends on the recording conditions, i.e., the conduction distance, the inter-electrode distance, and so on. Similarly, that of the FPs depends on the filter bandwidth and the tip diameter of the microelectrode.) Depending on the A/D card and filters used the optimal sampling rate could be determined from Equation (2) or Table 1. Although the required bandwidth of filtering and hence the optimal sampling rate depends on the origin or nature of the signal itself, the procedure used for its determination provides a tutorial and useful guide for other applications. Following this guideline one could be able to sample a signal without the contamination of aliasing crrors.

This work was supported by grants NSC89-2314-B-002-312-M08.

\section{References}

1. Freeman JA and Nicholson C. Experimental optimization of current source-density technique for anuran cerebellum. J Neurophysiol 1975; 38: 369-382.

2. Buzsaki G, Bickford RG, Ryan LJ, Young S, Prohaska O, Mandel RJ and Gage FH. Multisite recording of brain field potentials and unit activity in freely moving rats. J Neurosci Methods 1989; 28: 209-217.

3. Chen Y-Y. Current source density of ficld potential in the brain. M.S. thesis, Taipei, Natl. Taiwan Univ.; 1998.

4. Aldridge JW, Walden JL and Gilman S. Enhancing high-speed digitization of single-unit neuronal 
activity on a microcomputer using a hybrid software-hardware technique. J Neurosci Methods 1989; 28: 205-208.

5. Jaw F-S and Wang C-Y. Notes on the applications of A/D card for biomedical signals. Biomed Eng Appl Basis Comm 1993; 5: 773-777.

6. Stanley WD, Dougherty GR and Dougherty R. Digital signal processing. second ed., Reston Publishing; 1984.

7. Page RW and Foster AS. Waveform reconstruction techniques for precision digitizing oscilloscope. HP Journal 1988; Feb.: 26-31.

8. Cachelin AB, Dempster J and Gray pta. Computers. In: Ogden D editor. Microelectrode techniques, second ed., Cambridge, the Plymouth Workshop Handbook, The Company of Biologists Ltd.; 1994.

9. Jaw F-S, Yu SN, Lee J-C, Tsao H-W, Yu H-J and Yen C-T. Interactive program for spectral and area analysis of compound action potentials of A fiber and C fiber. J Neurosci Methods 1991; 40: 121-126.

10. Briano Jr. A reproducible technique for breaking glass micropipettes over a wide range of tip diameters. J Neurosci Methods 1983; 9: 31-34.

11. Siegel LC and Pearl RG. Pressure measurement artifact with analog-to-digital conversion. Journal of Clinical Monitoring 1990; 6: 318-321.

12. Turner DA and Schieckert M. Data acquisition and analysis system for intracellular neuronal signals. J. Neurosci. Methods 1990; 35: 241-251.

13. Gasser H S and Erlanger J. The action potential in fibers of slow conduction in spinal roots and somatic nerves. Am. J. Physiol. 1930; 92: 43-82.

14. Goovaerts HG and Rompelman O. Coherent average technique-a tutorial review. J Biomed Eng 1991; 13: 275-280. 